\title{
A comparative dosimetric study of volumetric-modulated arc therapy vs. fixed field intensity-modulated radiotherapy in postoperative irradiation of stage IB-IIA high-risk cervical cancer
}

\author{
LILI QIAO ${ }^{1}$, JIAN CHENG ${ }^{1}$, NING LIANG ${ }^{1}$, JIAN XIE $^{1}$, HUI LUO ${ }^{2}$ and JIANDONG ZHANG ${ }^{1}$ \\ ${ }^{1}$ Department of Radiation Oncology, Qianfoshan Hospital Affiliated to Shandong University, Jinan, Shandong 250014; \\ ${ }^{2}$ Department of Graduate, Weifang Medical University, Weifang, Shandong 261053, P.R. China
}

Received November 27, 2014; Accepted October 28, 2015

DOI: $10.3892 / \mathrm{ol} .2015 .3998$

\begin{abstract}
The aim of the present study was to compare the dosimetry features of volumetric-modulated arc therapy (VMAT) and fixed field intensity-modulated radiotherapy (f-IMRT) in postoperative irradiation of stage IB-IIA high-risk cervical cancer. Fifteen patients exhibiting stage IB-IIA high-risk cervical cancer, who had been treated with postoperative adjuvant concurrent radiochemotherapy, were selected. The clinical target volume (CTV) and organs at risk (OARs) were delineated according to contrast computed tomography images. The planning target volume (PTV) was subsequently produced by using $1 \mathrm{~cm}$ uniform expansion of the CTV. The treatment plans were intended to deliver $50 \mathrm{~Gy}$ in 25 fractions. The OARs that were contoured included the bladder, rectum, small bowel and femoral heads. Dose volume histograms were used to evaluate the dose distribution in the PTV and OARs. VMAT and f-IMRT treatment plans resulted in similar dose coverage of the PTV. VMAT was superior to f-IMRT in conformity $(\mathrm{P}<0.05)$, and resulted in a reduction of OARs irradiated at high dose levels (V40 and V50) compared with f-IMRT $(\mathrm{P}<0.05)$, particularly for the bladder. However, the doses of low levels (V10 and V20) delivered to OARs with f-IMRT were slightly reduced compared with VMAT $(\mathrm{P}<0.05)$. For ambilateral femoral heads, VMAT demonstrated improved sparing compared with f-IMRT, with regard to D5 $(\mathrm{P}<0.05)$. Furthermore, VMAT treatment plans revealed a significant reduction in monitor units (MU) and treatment time. VMAT techniques exhibited similar PTV coverage compared with f-IMRT. At doses of high levels delivered to OARs, VMAT demonstrated
\end{abstract}

Correspondence to: Dr Jiandong Zhang, Department of Radiation Oncology, Qianfoshan Hospital Affiliated to Shandong University, 16766 Jingshi Road, Jinan, Shandong 250014, P.R. China

E-mail: zhangjd165@sina.com

Key words: volumetric-modulated arc radiotherapy, cervical cancer, dosimetry characteristics, intensity-modulated radiotherapy improved sparing compared with f-IMRT, particularly for the bladder, while significantly reducing treatment time and MU number.

\section{Introduction}

Cervical cancer is the second most common type of cancer among women globally, particularly in developing countries $(1,2)$. Radical hysterectomy is the primary treatment for early-stage cervical cancer, and has demonstrated a 5-year overall survival rate of $80-90 \%(2,3)$. For patients exhibiting high-risk factors or locally advanced disease, postoperative adjuvant concurrent radiochemotherapy is typically recommended, which has been confirmed to reduce locoregional recurrence (4-7). However, there is a high incidence of radiation-induced toxicities of pelvic normal tissue, leading to a severely negative influence on patient quality of life (8). Furthermore, $\sim 30 \%$ of cervical cancer patients exhibiting stages IB2 to IV (International Federation of Gynecology and Obstetrics, FIGO) typically relapse following treatment $(8,9)$. In theory, a higher radiation dose directed at the target will reduce the likelihood of disease relapse, while potentially increasing the exposure of organs at risk (OARs), which in turn will limit the delivery of higher doses to the target. Therefore, it is important to utilize a treatment technique that is able to deliver an adequate dose to the tumor volume, while minimizing exposure of the OARs.

Intensity-modulated radiation therapy (IMRT) techniques have been widely utilized in a number of countries, and have produced improved dose distribution and OAR sparing compared with conventional radiotherapy, decreasing radiation-induced toxicity (10-12). Positive dose distribution and patient outcomes achieved by IMRT have been observed in several clinical studies (13-16). However, IMRT has additionally presented certain disadvantages, one of which is that the treatment delivery time is prolonged (17). Volumetric-modulated arc therapy (VMAT), a complex form of IMRT, has received considerable attention in the radiation oncology field, and has been investigated in a variety of malignant tumors, including esophageal, prostate and small brain tumors (17-19). Cozzi et al (20) reported that a RapidArc (RA) treatment plan for the treatment of cervical uterine cancer demonstrated 
improved sparing of OARs and healthy tissue, with uncompromising target coverage compared with fixed field (f)-IMRT. However, Zhai et al (21) concluded alternative results, demonstrating that f-IMRT possessed improved conformal planning target volume (PTV) coverage and OAR sparing, compared with VMAT, for the treatment of cervical cancer. Therefore, in the present study, a dosimetric comparison of VMAT and f-IMRT techniques was conducted in order to clarify whether VMAT possessed a dosimetric advantage in dose distribution of PTV and sparing of OARs compared with f-IMRT, thus achieving an optimization for the treatment planning for cervical cancer patients exhibiting stage IB-IIA high-risk cervical cancer following hysterectomy.

\section{Materials and methods}

Patients. A total of 15 patients (Karnofsky Performance Status; KPS $>70$ ) exhibiting stage IB-IIA cervical cancer with high-risk factors, who had received postoperative adjuvant concurrent radiochemotherapy (cisplatin-based chemotherapy and external beam radiotherapy for 45-50 Gy) between March and November 2012 at the Department of Radiation Oncology, Qianfoshan Hospital Affiliated to Shandong University (Jinan, China), were selected for the present study. According to FIGO, the clinical stages of all patients were as follows: 9 patients were stage IB and 6 were stage IIA. All patients underwent radical hysterectomy and pelvic lymph node dissection. The high-risk factors included pelvic lymph node metastases, parametrial invasion and positive surgical margins. Patient ages ranged from 34-59 years, with a median age of 43 years. Detailed patient information is listed in Table I.

The present study was approved by the Qianfoshan Hospital Ethics Committee, and informed consent was obtained from all patients.

Target volume and organs at risk delineation. All patients were immobilized in a supine position with bladder filling, and stimulated utilizing a computed tomography (CT) scanner with a $3 \mathrm{~mm}$ slice thickness from the superior margin of the L4 to the ischial tuberosities. According to the consensus guidelines of Taylor et al (22), the target volumes were delineated by an experienced radiation oncologist and radiologist. The clinical target volume (CTV) included the upper $3.0 \mathrm{~cm}$ of the vagina, paravaginal soft tissue lateral to the vagina and pelvic lymph node regions (the common iliac, external and internal iliac, and presacral lymph nodes). The PTV was generated by using $1 \mathrm{~cm}$ uniform expansion of the CTV in all directions, based on the guidelines set out by Small et al (23). The OARs that were contoured comprised the bladder, rectum, small bowel and femoral heads. The rectum was defined from the level of the rectosigmoid junction to the anus. The bladder was contoured entirely. The small bowel was contoured from the interspace of L4-5 to its lowest extent in the pelvis. The femoral heads were delineated from the cranial end point to the lower margin of the ischial tuberosities.

Dose prescription and constraints. VMAT and f-IMRT treatment plans were designed for a treatment in 25 fractions, with the aim of delivering a total dose of 50 Gy to the PTV,
Table I. Patient characteristics.

\begin{tabular}{lc}
\hline Variable & Value \\
\hline Age, years & \\
Mean & 43 \\
Range & $34-59$ \\
Stage ${ }^{\mathrm{a}}$, number of patients & \\
IB & 9 \\
IIA & 6 \\
Histology, number of patients & \\
Squamous carcinoma & 10 \\
Adenocarcinoma & 5 \\
\hline
\end{tabular}

${ }^{a}$ According to the International Federation of Gynecology and Obstetrics.

using the Eclipse ${ }^{\text {TM }}$ Treatment Planning System (Version 10.0; Varian Medical Systems, Inc., Palo Alto, CA, USA), with a $6 \mathrm{MV}$ beam modulator linear accelerator (TrueBeam; Varian Medical Systems, Inc.). The treatment plans aimed to cover $\geq 95 \%$ of the volume of PTV with the prescribed dose, and to restrict a mean dose of $<105 \%$ and a maximum dose of $<110 \%$ of the prescribed dose. The dose delivered to $50 \%$ of the tumor volume was $<45 \mathrm{~Gy}$ for the bladder, and $50 \mathrm{~Gy}$ for the rectum and the small bowel. For the femoral heads, the dose delivered to $5 \%$ of the tumor volume was $<45 \mathrm{~Gy}$.

IMRT and VMAT treatment plans. f-IMRT treatment plans were optimized with a Dose Volume Optimizer engine (version 10.0.28; Varian Medical Systems, Palo Alto, CA, USA), and dose calculation was performed by anisotropic analytical algorithm. The f-IMRT treatment plans were performed using five coplanar fields $(5 \mathrm{~F})$ with the dynamic sliding window technique, as 5F-IMRT is widely used as a treatment choice for eligible patients at Qianfoshan Hospital. Each treatment field constituted beam geometry, with the following gantry angles: $0^{\circ} / 72^{\circ} / 144^{\circ} / 216^{\circ} / 288^{\circ}$.

The VMAT treatment plans were optimized with the Progressive Resolution Optimizer (PRO; version 10.0.28; Varian Medical Systems, Palo Alto, CA, USA). Two full arcs were used in the VMAT treatment plans, which consisted of two coplanar $\operatorname{arcs}$ of $360^{\circ}$ with opposite rotation. The first arc rotated clockwise for $358^{\circ}$ and started with a gantry angle of $181^{\circ}$ to stop at $179^{\circ}$, while the second arc rotated anticlockwise from the gantry angle of $179^{\circ}$ to $181^{\circ}$. The couch was set to $0^{\circ}$, and the collimator angle was set to $30^{\circ}$ (or $330^{\circ}$ ) to avoid tongue-and-groove effects. Each arc was limited to a sequence of 178 control points. Two coplanar arcs allowed superior modulation factors during optimization, which contributed to acquiring higher target homogeneity and improved OAR sparing.

VMAT and f-IMRT plans were exported by Digital Imaging and Communication in Medicine (DICOM; Varian Medical Systems, Palo Alto, CA, USA) radiotherapy export.

Evaluation parameters. Data from the dose volume histograms acquired for each patient were analyzed. For the 
target-dose coverage, a set of dosimetric parameters was analyzed, including the mean dose (Dmean), maximum dose, V95\% (i.e. the percent of the tumor volume that received $\mathrm{x} \%$ of the prescribed dose), V105\%, conformity index (CI) and homogeneity index (HI).

$\mathrm{CI}$, as a means to assess the conformity of dose distribution, was calculated as follows:

$$
C I=\frac{P T V 100}{P T V} \times \frac{P T V 100}{V 100}
$$

In which, PTV100 was the target volume covered by the prescribed dose, PTV was the target volume and V100 was the volume of the prescribed dose. Higher values (close to 1) of CI represented an improved PTV conformity. HI was defined as follows:

$$
H I=\frac{D 5 \%}{D 95 \%}
$$

In which, Dx\% represented the dose delivered to $\mathrm{x} \%$ of the PTV. Lower values (close to 1) of HI indicated a more homogenous PTV dose distribution.

To investigate the evaluation parameters of OARs, for bladder, rectum and small bowel, a series of $\mathrm{Vx}$ values were analyzed respectively, and for femoral heads, the D5 from the two treatment plans were compared.

For each patient, treatment time and the number of MUs were evaluated. Patient positioning, gantry and collimator rotation times were excluded when treatment time was recorded.

Statistical analysis. All statistical analyses of VMAT and f-IMRT treatment plans were performed using SPSS (Version 13.0; SPSS, Inc., Chicago, IL, USA). Paired sample t-test was utilized for the calculation of statistical difference. $\mathrm{P}<0.05$ was considered to indicate a statistically significant difference.

\section{Results}

Target-dose coverage is slightly reduced in VMAT compared with $f$-IMRT. VMAT and f-IMRT treatment plans of all 15 patients were completed according to the aforementioned design. The dosimetric parameters associated with the target of the two treatment plans are presented in Table II. The dose distribution of VMAT and f-IMRT treatment plans in a single representative patient is revealed in Fig. 1. The results of the present study indicated that VMAT demonstrated an equivalent dose coverage to the target compared with f-IMRT.

The maximum dose to the PTV was slightly increased for f-IMRT compared with VMAT, but no significant difference was observed between the two treatment plans $(\mathrm{P}>0.05)$. Regarding the comparison of Dmean, a similar trend was identified $(51.06 \pm 0.14$ Gy for f-IMRT vs. 50.93 \pm 0.24 Gy for VMAT; $\mathrm{P}=0.295)$. There was no significant difference between the two plans in the comparison of $\mathrm{V} 95 \%$ and $\mathrm{V} 105 \%$ $(\mathrm{P}>0.05)$.

Conformity was improved in VMAT compared with f-IMRT, and a statistically significant difference was identified $(0.82 \pm 0.01$ vs. $0.78 \pm 0.04$, respectively; $\mathrm{P}<0.05)$. However, there was no significant difference between VMAT and f-IMRT in terms of HI $(1.03 \pm 0.08$ vs. $1.04 \pm 0.01 ; \mathrm{P}>0.05)$.
Table II. Comparison of the dosimetric parameters of the planning target volume, MU and treatment time.

\begin{tabular}{lccc}
\hline Variable & IMRT & VMAT & P-value \\
\hline Dmax & $52.97 \pm 0.26$ & $52.62 \pm 0.32$ & 0.219 \\
Dmean & $51.06 \pm 0.14$ & $50.93 \pm 0.24$ & 0.295 \\
V95\% & $99.86 \pm 0.06$ & $99.96 \pm 0.03$ & 0.739 \\
V105\% & $0.33 \pm 0.47$ & $0.24 \pm 0.42$ & 0.342 \\
CI & $0.78 \pm 0.04$ & $0.82 \pm 0.01$ & 0.010 \\
HI & $1.04 \pm 0.01$ & $1.03 \pm 0.08$ & 0.718 \\
MU & $1147.38 \pm 107.34$ & $377.75 \pm 45.83$ & 0.946 \\
Time & $2.87 \pm 0.27$ & $1.06 \pm 0.14$ & 0.552 \\
\hline
\end{tabular}

IMRT, intensity-modulated radiotherapy; VMAT, volumetric-modulated arc therapy; Dmax, maximal dose; Dmean, mean dose; Vx\%, target tumor volume covered by $\mathrm{X} \%$ of the prescribed dose; CI, conformity index; HI, homogeneity index; MU, monitor unit.

Table III. Comparison of the dosimetric parameters of the organs at risk.

\begin{tabular}{lccc}
\hline Variable & IMRT & VMAT & P-value \\
\hline Bladder, \% & & & \\
V10 & $99.96 \pm 0.11$ & $100.00 \pm 0.00$ & 0.344 \\
V20 & $97.62 \pm 5.09$ & $99.79 \pm 0.49$ & 0.000 \\
V30 & $84.32 \pm 8.64$ & $84.42 \pm 14.89$ & 0.003 \\
V40 & $55.30 \pm 9.38$ & $51.40 \pm 8.90$ & 0.199 \\
V50 & $21.79 \pm 5.71$ & $17.82 \pm 7.15$ & 0.012 \\
Rectum, \% & & & \\
V10 & $97.92 \pm 3.38$ & $98.34 \pm 2.78$ & 0.012 \\
V20 & $96.65 \pm 3.90$ & $97.52 \pm 2.57$ & 0.063 \\
V30 & $88.45 \pm 9.82$ & $91.87 \pm 7.99$ & 0.000 \\
V40 & $66.65 \pm 13.41$ & $65.34 \pm 13.30$ & 0.000 \\
V50 & $22.08 \pm 7.58$ & $22.37 \pm 8.30$ & 0.000 \\
Small bowel, \% & & & \\
V10 & $78.53 \pm 14.30$ & $80.27 \pm 23.14$ & 0.003 \\
V20 & $57.78 \pm 16.77$ & $56.59 \pm 18.69$ & 0.001 \\
V30 & $38.22 \pm 11.89$ & $34.64 \pm 12.54$ & 0.000 \\
V40 & $20.65 \pm 8.79$ & $19.42 \pm 11.87$ & 0.000 \\
V50 & $7.81 \pm 5.25$ & $7.40 \pm 5.40$ & 0.000 \\
Left femoral head, Gy & & & \\
D5 & $38.26 \pm 4.35$ & $37.80 \pm 4.36$ & 0.000 \\
Right femoral head, Gy & & & \\
D5 & $37.27 \pm 4.49$ & $36.13 \pm 4.35$ & 0.005 \\
& & & \\
\hline
\end{tabular}

IMRT, intensity-modulated radiotherapy; VMAT, volumetric-modulated arc therapy; Vx, target tumor volume covered by X Gy; D5, dose delivered to $5 \%$ of the femoral head.

OAR sparing is largely superior in VMAT. Parameters associated with the dose delivered to OARs for VMAT and f-IMRT are revealed in Table III. Fig. 2 illustrates the differences between the VMAT and f-IMRT treatment plans in a single representative patient. 

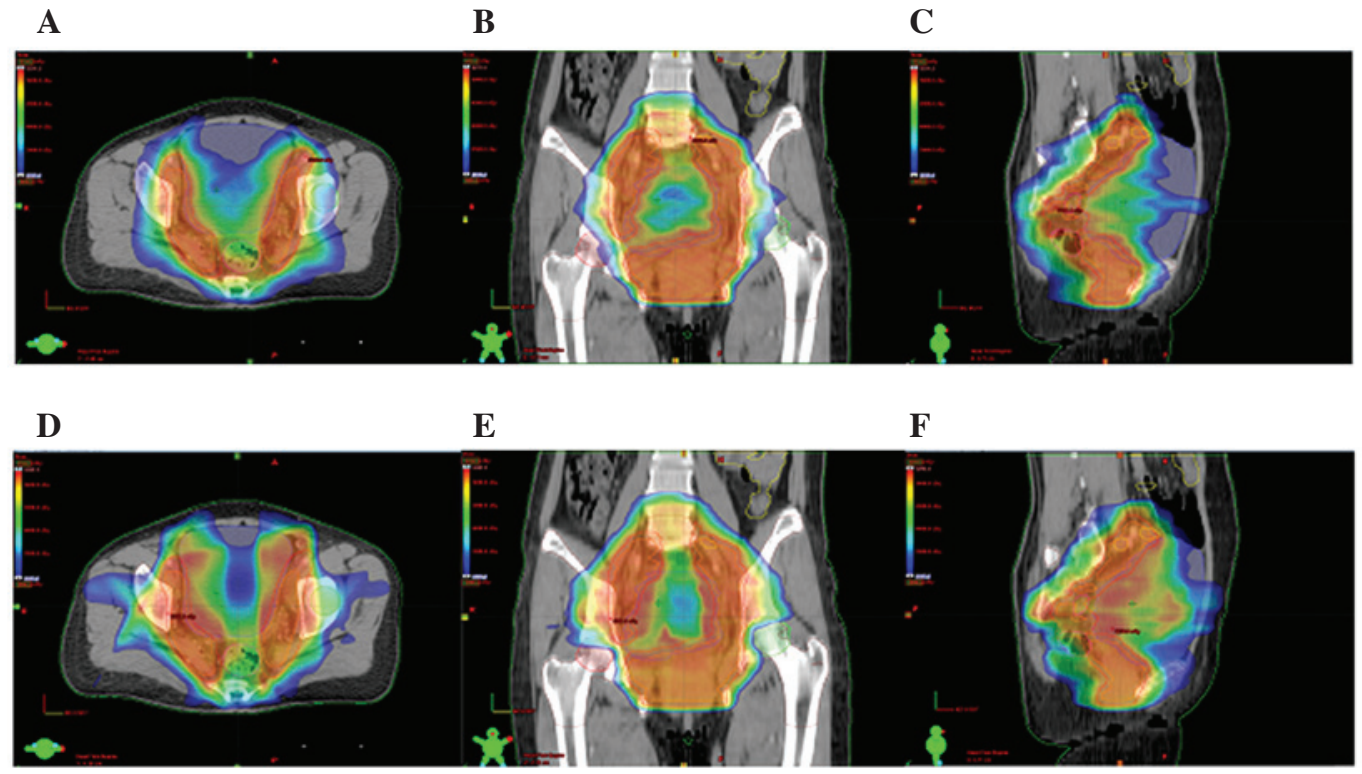

Figure 1. Dose distributions of the VMAT and IMRT plans in a single representative patient by horizontal view (A and D), coronal view (B and E) and sagittal view (C and F). (A-C) VMAT dose distribution. (D-F) IMRT dose distribution. Red line, planning target volume; Orange line, clinical target volume. VMAT, volumetric-modulated arc therapy; IMRT, intensity-modulated radiotherapy.

For the bladder, f-IMRT was superior to VMAT for OAR sparing at V20 and V30, and this difference was statistically significant $(\mathrm{P}<0.05)$. However, the advantage for f-IMRT was not observed with regard to V40 $(55.30 \pm 9.38 \%$ for f-IMRT vs. $51.40 \pm 8.90 \%$ for VMAT; P>0.05). V50 for f-IMRT was, on average, higher than VMAT, with a statistically significant difference $(21.79 \pm 5.71 \%$ for f-IMRT vs. $17.82 \pm 7.15 \%$ for VMAT; $\mathrm{P}<0.05)$. For the rectum, a similar trend of dose distribution (V10, V20 and V30) was observed between the two techniques (V10, $\mathrm{P}=0.012 ; \mathrm{V} 20, \mathrm{P}=0.063$; V30, $\mathrm{P}<0.0001$ ). Compared with f-IMRT, V40 was slightly lower in VMAT (65.34 \pm 13.30 vs. $66.65 \pm 13.41 \%$; $\mathrm{P}<0.05)$. A statistically significant difference was observed for V50 between the VMAT and f-IMRT treatment plans $(22.37 \pm 8.30$ vs. $22.08 \pm 7.58 \%$; $\mathrm{P}<0.05)$. For the small bowel, V10 in f-IMRT was slightly lower compared with that observed in VMAT (78.53 \pm 14.30 vs. $80.27 \pm 23.14 \%$; $\mathrm{P}<0.05)$. However, an adverse trend was observed in terms of V20, V30, V40 and V50, and VMAT was slightly improved compared with f-IMRT for these parameters $(\mathrm{P}<0.05)$. For ambilateral femoral heads, VMAT demonstrated a slight advantage compared with f-IMRT $(\mathrm{P}<0.05)$.

Treatment time and monitor units are reduced in VMAT. The treatment time by VMAT was $1.06 \mathrm{~min}$, which was 2.7 times shorter compared with f-IMRT (1.06 \pm 0.14 vs. $2.87 \pm 0.27 \mathrm{~min})$. For MU, a significant reduction was observed for VMAT compared with f-IMRT (377.75 \pm 45.83 vs. $1,147.38 \pm 107.34)$. Details are presented in Table II.

\section{Discussion}

In the present study, a series of dosimetric parameters indicated that VMAT and f-IMRT treatment plans were able to meet the requirements for target coverage and OAR sparing.
Furthermore, VMAT exhibited a similar or improved dose distribution to the target and sparing of OARs compared with f-IMRT, and significantly reduced the treatment time and MU number. Similar results were observed by Cozzi et al (20) and Palma et al (24). However, the results of the present study were not consistent with those of Zhai et al (21) and Yoo et al (25), in which f-IMRT demonstrated improved conformal PTV coverage and OAR sparing compared with RA. This difference may be due to the discrepancy between target volume delineation and treatment plan formulations.

VMAT, as a rotational form of IMRT, enables dose delivery primarily in single or double arcs (20). In the present study, dual arcs were utilized, which have resulted in superior modulation factor during optimization and highly conformal dose by varying the speed of gantry rotation, the multileaf collimator shape and the dose rate. Verbakel et al (26) demonstrated that dual-arc VMAT treatment plans produced similar or improved OAR sparing, and superior target dose homogeneity, compared with single-arc VMAT in head and neck cancer. In addition, a study performed by Guckenberger et al (27) indicated that dual-arc VMAT may improve target coverage and dose homogeneity compared with single-arc VMAT. Furthermore, considering the complexity of the target volume and importance of critical organ sparing, the present study selected dual-arc VMAT techniques that yielded a promising and clinically acceptable outcome for the current experiments.

Comparing VMAT with f-IMRT, the present study identified that the two techniques met the requirements of clinical treatment for PTV coverage. VMAT proved to be superior to f-IMRT in terms of CI, however, for HI this advantage was not observed. For the bladder, rectum and small bowel, f-IMRT was similar or slightly improved compared with VMAT at low dose levels of V10, V20 and V30. However, VMAT proved to be superior to f-IMRT at high dose levels of V40 or V50, 


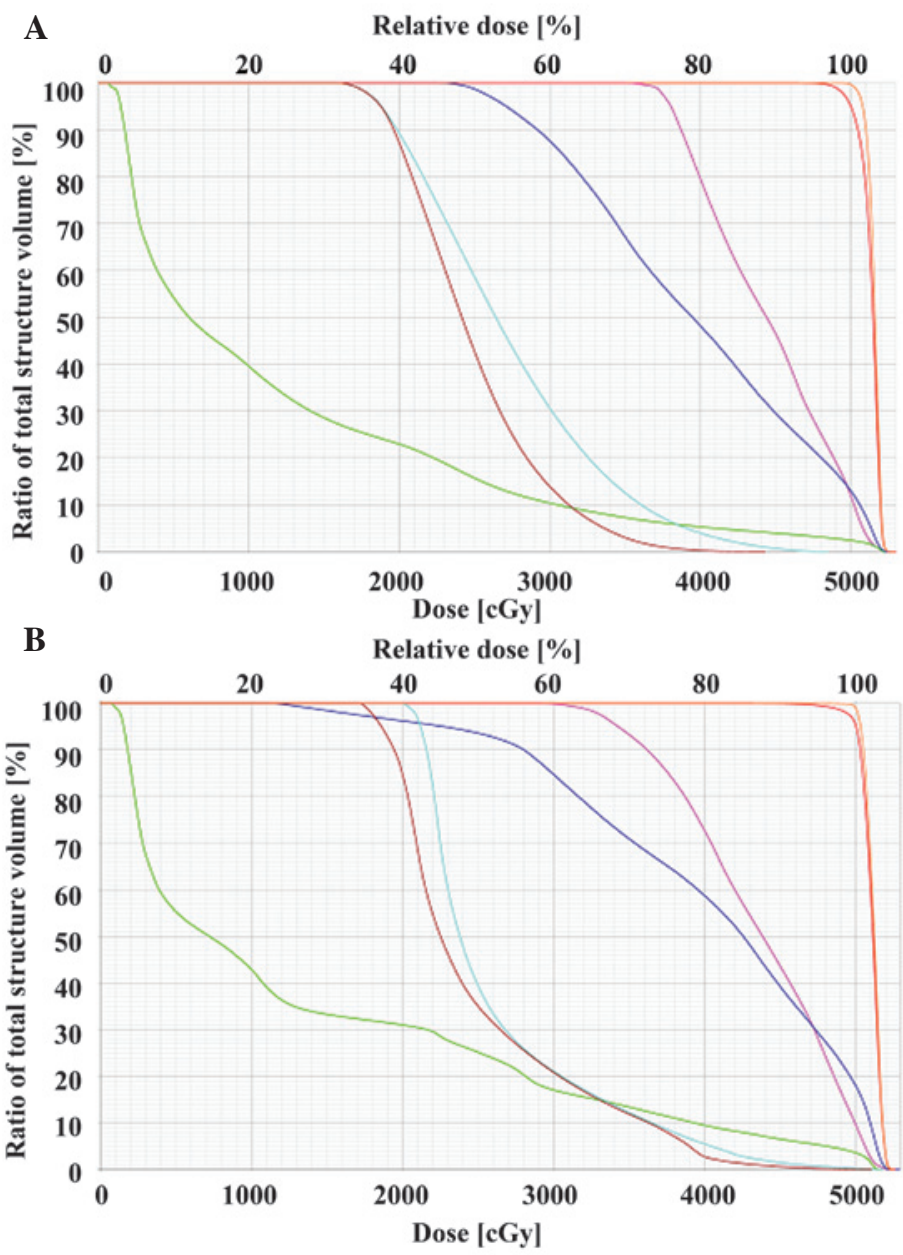

Figure 2. Dose-volume histogram of a representative patient for VMAT and IMRT. (A) VMAT, (B) IMRT. Red line (-), planning target volume; Orange line (-), clinical target volume; Dark blue line (-), bladder; Magenta line (-), rectum; Yellow line (-), small bowel; Cyan line (-), left femur; Brown line (-), right femur. VMAT, volumetric-modulated arc therapy; IMRT, intensity-modulated radiotherapy.

particularly for the bladder. Therefore, VMAT may be preferentially used for the treatment of patients exhibiting cervical cancer with accompanying bladder disease, to improve bladder sparing. However, whether VMAT is able to reduce radiation-induced bladder injury or bring long-term clinical benefit remains to be elucidated, and requires further investigation by means of clinical studies. With regard to femoral heads, VMAT provided improved sparing by reducing the delivery dose compared with f-IMRT.

f-IMRT demonstrated an additional number of MUs compared with VMAT. The treatment time for f-IMRT was 2.7 times longer than that for VMAT. The results of the present study suggested that VMAT may significantly reduce treatment time and MU number compared with f-IMRT, which may decrease the effects of intrafractional motion due to patient movement occurring during treatment delivery (28). Furthermore, the reduction in treatment time was capable of lessening the radiation effects to critical structures, which were adjacent to the target tumor volume (29), and the reduction of MU may decrease the number of radiation-induced secondary cancers by reducing leakage radiation (30). In addition, Wang et al (31) demonstrated that prolonged delivery time of $>10-15$ min may reduce tumor cell killing, due to increased sublethal damage repair. Moiseenko et al (32) investigated cell survival for three cervical carcinoma cell lines, which were irradiated for $75 \mathrm{~s}$, $5 \mathrm{~min}$ and $10 \mathrm{~min}$ respectively, and identified that the survival rates of the three cell lines were 39, 53 and $59 \%$, respectively. The above-mentioned studies indicate that short treatment time and reduced MU numbers contribute to the acquisition of a superior clinical outcome for local tumor control. Consequently, in the present study improved tumor control was observed following treatment with VMAT. However, the planning time used for VMAT was markedly increased compared with f-IMRT, as the computational speed of the optimization process was slower in VMAT than in f-IMRT for the planning systems utilized in the present study. However, when utilizing certain automatic scripts set up in advance, each plan may be completed in a short time.

The results of the present study may be influenced by the following limitations. Intrafractional organ motion may not have been detected due to an absence of a cone beam CT facility in the department, which may have influenced the results of dose delivery to the target and OARs. In addition, patient positioning and bladder filling status were not monitored due to a lack of image guidance. Furthermore, the present study was limited by a small sample size, which negated the precise evaluation of the dosimetric parameters between the two treatment plans. 
Based on the results of the present study, it was concluded that in stage IB-IIA high-risk cervical cancer irradiation, the VMAT technique demonstrated certain advantages, not in superior dose distribution or OAR sparing, but in short treatment delivery time and low MU number. With additional performance of clinical studies, the advantages of VMAT techniques may be confirmed.

In the present study, VMAT and f-IMRT techniques met the clinical requirements for dose distribution and OAR sparing. VMAT techniques proved to generate similar PTV coverage compared with f-IMRT. However, VMAT led to a reduction of OAR volumes irradiated at high dose levels in comparison with f-IMRT, particularly for the bladder, significantly reducing the treatment time and MU number.

\section{Acknowledgements}

The present study was supported by the Natural Science Foundation of Shandong Province (grant no. ZR2012HM095).

\section{References}

1. Jemal A, Siegel R, Xu J and Ward E: Cancer statistics, 2010. CA Cancer J Clin 60: 277-300, 2010.

2. Kim JH, Choi JH, Ki EY, Lee SJ, Yoon JH, Lee KH, Park TC, Park JS, Bae SN and Hur SY: Incidence and risk factors of lower-extremity lymphedema after radical surgery with or without adjuvant radiotherapy in patients with FIGO stage I to stage IIA cervical cancer. Int J Gynecol Cancer 22: 686-691, 2012.

3. Landoni F, Maneo A, Colombo A, Placa F, Milani R, Perego P, Favini G, Ferri L and Mangioni C: Randomised study of radical surgery versus radiotherapy for stage Ib-IIa cervical cancer. Lancet 350: 535-540, 1997.

4. Mabuchi S, Morishige K, Isohashi F, Yoshioka Y, Takeda T, Yamamoto T, Yoshino K, Enomoto T, Inoue T and Kimura T: Postoperative concurrent nedaplatin-based chemoradiotherapy improves survival in early-stage cervical cancer patients with adverse risk factors. Gynecol Oncol 115: 482-487, 2009.

5. Monk BJ, Tewari KS and Koh WJ: Multimodality therapy for locally advanced cervical carcinoma: State of the art and future directions. J Clin Oncol 25: 2952-2965, 2007.

6. Sehouli J, Runnebaum IB, Fotopoulou C, Blohmer U, Belau A, Leber H, Hanker LC, Hartmann W, Richter R, Keyver-Paik MD, et al: A randomized phase III adjuvant study in high-risk cervical cancer: Simultaneous radiochemotherapy with cisplatin (S-RC) versus systemic paclitaxel and carboplatin followed by percutaneous radiation (PC-R): A NOGGO-AGO intergroup study. Ann Oncol 23: 2259-2264, 2012.

7. Zhang G, Fu C, Zhang Y, Wang J, Qiao N, Yang Q and Cheng Y: Extended-field intensity-modulated radiotherapy and concurrent cisplatin-based chemotherapy for postoperative cervical cancer with common iliac or para-aortic lymph node metastases: A retrospective review in a single institution. Int J Gynecol Cancer 22: 1220-1225, 2012

8. Grigsby PW and Perez CA: Radiotherapy alone for medically inoperable carcinoma of the cervix: Stage IA and carcinoma in situ. Int J Radiat Oncol Biol Phys 21: 375-378, 1991.

9. Du XL, Tao J, Sheng XG, Lu CH, Yu H, Wang C, Song QQ, Li QS and Pan CX: Intensity-modulated radiation therapy for advanced cervical cancer: A comparison of dosimetric and clinical outcomes with conventional radiotherapy. Gynecol Oncol 125: 151-157, 2012.

10. Randall ME and Ibbott GS: Intensity-modulated radiation therapy for gynecologic cancers: Pitfalls, hazards, and cautions to be considered. Semin Radiat Oncol 16: 138-143, 2006.

11. Vergeer MR, Doornaert PA, Rietveld DH, Leemans CR, Slotman BJ and Langendijk JA: Intensity-modulated radiotherapy reduces radiation-induced morbidity and improves health-related quality of life: Results of a nonrandomized prospective study using a standardized follow-up program. Int J Radiat Oncol Biol Phys 74: 1-8, 2009.

12. van Rij CM, Oughlane-Heemsbergen WD, Ackerstaff AH, Lamers EA, Balm AJ and Rasch CR: Parotid gland sparing IMRT for head and neck cancer improves xerostomia related quality of life. Radiat Oncol 3: 41, 2008.
13. Rochet N, Kieser M, Sterzing F, Krause S, Lindel K, Harms W, Eichbaum MH, Schneeweiss A, Sohn C and Debus J: Phase II study evaluating consolidation whole abdominal intensity-modulated radiotherapy (IMRT) in patients with advanced ovarian cancer stage FIGO III - the OVAR-IMRT-02 Study. BMC Cancer 11: 41, 2011.

14. Dirix P, Vanstraelen B, Jorissen M, Vander Poorten V and Nuyts S: Intensity-modulated radiotherapy for sinonasal cancer: Improved outcome compared to conventional radiotherapy. Int J Radiat Oncol Biol Phys 78: 998-1004, 2010.

15. Staffurth J; Radiotherapy Development Board: A review of the clinical evidence for intensity-modulated radiotherapy. Clin Oncol (R Coll Radiol) 22: 643-657, 2010.

16. Pederson AW, Fricano J, Correa D, Pelizzari CA and Liauw SL: Late toxicity after intensity-modulated radiation therapy for localized prostate cancer: An exploration of dose-volume histogram parameters to limit genitourinary and gastrointestinal toxicity. Int J Radiat Oncol Biol Phys 82: 235-241, 2012.

17. Wolff D, Stieler F, Welzel G, Lorenz F, Abo-Madyan Y, Mai S, Herskind C, Polednik M, Steil V, Wenz F and Lohr F: Volumetric modulated arc therapy (VMAT) vs. serial tomotherapy, step-and-shoot IMRT and 3D-conformal RT for treatment of prostate cancer. Radiother Oncol 93: 226-233, 2009.

18. Mayo CS, Ding L, Addesa A, Fitzgerald TJ and Moser R: Initial experience with volumetric IMRT (RapidArc) for intracranial stereotactic radiosurgery. Int J Radiat Oncol Biol Phys 78: 1457-1466, 2010.

19. Yin L, Wu H, Gong J, Geng JH, Jiang F, Shi AH, Yu R, Li YH, Han SK, Xu B and Zhu GY: Volumetric-modulated arc therapy vs. c-IMRT in esophageal cancer: A treatment planning comparison. World J Gastroenterol 18: 5266-5275, 2012.

20. Cozzi L, Dinshaw KA, Shrivastava SK, Mahantshetty U, Engineer R, Deshpande DD, Jamema SV, Vanetti E, Clivio A, Nicolini G and Fogliata A: A treatment planning study comparing volumetric arc modulation with RapidArc and fixed field IMRT for cervix uteri radiotherapy. Radiother Oncol 89: 180-191, 2008.

21. Zhai DY, Yin Y, Gong GZ, Liu TH, Chen JH, Ma CS and Lu J: RapidArc radiotherapy for whole pelvic lymph node in cervical cancer with 6 and $15 \mathrm{MV}$ : A treatment planning comparison with fixed field IMRT. J Radiat Res 54: 166-173, 2013.

22. Taylor A, Rockall AG, Reznek RH and Powell ME: Mapping pelvic lymph nodes: Guidelines for delineation in intensity-modulated radiotherapy. Int J Radiat Oncol Biol Phys 63: 1604-1612, 2005.

23. Small W Jr, Mell LK, Anderson P, Creutzberg C, De Los Santos J, Gaffney D, Jhingran A, Portelance L, Schefter T, Iyer R, et al: Consensus guidelines for delineation of clinical target volume for intensity-modulated pelvic radiotherapy in postoperative treatment of endometrial and cervical cancer. Int J Radiat Oncol Biol Phys 71: 428-434, 2008.

24. Palma D, Vollans E, James K, Nakano S, Moiseenko V, Shaffer R, McKenzie M, Morris J and Otto K: Volumetric modulated arc therapy for delivery of prostate radiotherapy: Comparison with intensity-modulated radiotherapy and three-dimensional conformal radiotherapy. Int J Radiat Oncol Biol Phys 72: 996-1001, 2008.

25. Yoo S, Wu QJ, Lee WR and Yin FF: Radiotherapy treatment plans with RapidArc for prostate cancer involving seminal vesicles and lymph nodes. Int J Radiat Oncol Biol Phys 76: 935-942, 2010.

26. Verbakel WF, Cuijpers JP, Hoffmans D, Bieker M, Slotman BJ and Senan S: Volumetric intensity-modulated arc therapy vs. conventional IMRT in head-and-neck cancer: A comparative planning and dosimetric study. Int J Radiat Oncol Biol Phys 74: 252-259, 2009.

27. Guckenberger M, Richter A, Krieger T, Wilbert J, Baier K and Flentje M: Is a single arc sufficient in volumetric-modulated arc therapy (VMAT) for complex-shaped target volumes? Radiother Oncol 93: 259-265, 2009.

28. Nguyen K, Cummings D, Lanza VC, Morris K, Wang C, Sutton J and Garcia J: A dosimetric comparative study: Volumetric modulated arc therapy vs intensity-modulated radiation therapy in the treatment of nasal cavity carcinomas. Med Dosim 38: 225-232, 2013.

29. Rao M, Yang W, Chen F, Sheng K, Ye J, Mehta V, Shepard D and Cao D: Comparison of Elekta VMAT with helical tomotherapy and fixed field IMRT: Plan quality, delivery efficiency and accuracy. Med Phys 37: 1350-1359, 2010.

30. Hall EJ: Intensity-modulated radiation therapy, protons, and the risk of second cancers. Int J Radiat Oncol Biol Phys 65: 1-7, 2006.

31. Wang JZ,Li XA, D'Souza WD and Stewart RD: Impact of prolonged fraction delivery times on tumor control: A note of caution for intensity-modulated radiation therapy (IMRT). Int J Radiat Oncol Biol Phys 57: 543-552, 2003.

32. Moiseenko V, Duzenli C and Durand RE: In vitro study of cell survival following dynamic MLC intensity-modulated radiation therapy dose delivery. Med Phys 34: 1514-1520, 2007. 